\title{
Review Article \\ Inflammation and Immunity in Radiation Damage to the Gut Mucosa
}

\author{
Agnès François, Fabien Milliat, Olivier Guipaud, and Marc Benderitter \\ Laboratory of Radiopathology and Experimental Therapeutics, Institute for Radiological Protection and Nuclear Safety, \\ 31 Avenue de la Division Leclerc, 92262 Fontenay-aux-Roses, France
}

Correspondence should be addressed to Agnès François; agnes.francois@irsn.fr

Received 30 October 2012; Accepted 18 February 2013

Academic Editor: Silvia Gregori

Copyright (C) 2013 Agnès François et al. This is an open access article distributed under the Creative Commons Attribution License, which permits unrestricted use, distribution, and reproduction in any medium, provided the original work is properly cited.

\begin{abstract}
Erythema was observed on the skin of the first patients treated with radiation therapy. It is in particular to reduce this erythema, one feature of tissue inflammation, that prescribed dose to the tumor site started to be fractionated. It is now well known that radiation exposure of normal tissues generates a sustained and apparently uncontrolled inflammatory process. Radiation-induced inflammation is always observed, often described, sometimes partly explained, but still today far from being completely understood. The thing with the gut and especially the gut mucosa is that it is at the frontier between the external milieu and the organism, is in contact with a plethora of commensal and foreign antigens, possesses a dense-associated lymphoid tissue, and is particularly radiation sensitive because of a high mucosal turnover rate. All these characteristics make the gut mucosa a strong responsive organ in terms of radiation-induced immunoinflammation. This paper will focus on what has been observed in the normal gut and what remains to be done concerning the immunoinflammatory response following localized radiation exposure.
\end{abstract}

\section{Introduction}

The objective of radiation therapy is to deliver a dose of ionizing radiations sufficient to ensure tumor control and to avoid cancer recurrence. The treatment of malignant tumors by radiation therapy is, however, invariably associated with radiation exposure of surrounding healthy tissues and the development of acute injury followed by late structural and/or functional damage, directly or indirectly linked to the initial trauma. Increased tumor control efficiency and life expectancy augment the risk to develop radiation sequels in patients in whom cure has been achieved.

\section{Pelvic Radiation Therapy and "Pelvic Radiation Disease"}

Intestinal tissue is particularly radiosensitive and remains the limiting factor in the application of radiotherapeutic schedules for the treatment of tumors located in the pelvis area. Treatment efficacy relies on a compromise between the quality of tumor control achieved by radiation therapy and the damages generated on intestinal healthy tissues; this is referred to as the benefit/risk ratio [1]. The definition of the irradiation field is governed by several factors that illustrate the necessity to include a proportion of normal tissues: possible tumor extensions undetected by medical imaging techniques, uncertainty concerning patient positioning reproducibility between each fraction, and the mobility of tumor and healthy organs during and between each fraction. Fractionated irradiation reduces the probability of high-dose exposure for mobile parts of the digestive tract, such as the small intestine. Conversely, the risk is increased for fixed segments such as the terminal ileum, colon, or rectum which are often concerned in irradiation protocols for cervical, endometrial, rectal, and prostatic cancers treatments. One strategy to limit normal tissue radiation exposure was to improve radiation therapy techniques and tumor imaging. Thus, precise tumor delineation and the use of three dimensions Conformal Radiation Therapy (3D-CRT) and IntensityModulated Radiation Therapy (IM-RT) reduce normal tissue volume located in the irradiation field and consequently treatment side effects.

Radiation gastrointestinal toxicity concerns the majority of patients treated for pelvic cancers. Acute symptoms are 
declared during or shortly after the end of the radiation therapy and are characterized by abdominal pain, diarrhoea and incontinency, less frequently constipation, bleeding, and mucus discharge. Patient's clinical status sometimes evolves through an aggravation of the acute symptoms, with diarrhoea alternately with constipation, severe abdominal pain, and sometimes nausea and vomiting. Clinical expression of gastrointestinal radiation toxicity often resembles other pathologies such as Crohn's disease, ulcerative colitis, or celiac disease, complicating the diagnosis especially when there has been a long period of time between radiation therapy and clinical symptoms expression. To improve the diagnosis, consideration, and management of the gastrointestinal consequences of radiation therapy of pelvic cancers, ranging from minor changes in bowel habits to severe painful and life-strengthening gastrointestinal dysfunction, the term of "pelvic radiation disease" has been suggested recently by Andreyev et al. [2,3].

\section{Radiation-Induced Breakdown of the Fragile Balance Governing Mucosal Homeostasis}

The intestine is a hierarchised self-renewing tissue, the entire mucosa being replaced every 3-5 days. Cell production is assumed by stem cells residing at the crypt bottom. The number of stem cells is estimated between 4 to 6 cells per crypt [4]. Daughter cells exit the stem cell compartment, reaching the transit amplifying compartment in which they achieve several divisions and are also named as committed precursors or progenitors cells. New produced cells then migrate along the crypt/villus axis and differentiate into 3 cell lineages of the intestinal mucosa, that is, epithelial bordering cells, goblet cells, and enteroendocrine cells. The fourth cell lineage, the Paneth cells, also derives from stem cells but migrates toward the bottom of the crypts.

Ionizing radiations are used to cure cancers based on their properties to kill cells by energy deposition in tissues, water radiolysis, and production of free radicals damaging DNA, proteins and lipids. Radiation-induced molecular damage on DNA can induce cell phenotypic modifications and/or death by apoptosis, necrosis, or mitotic catastrophe. Indirect biological effects consist in cell water radiolysis and generation of a burst of free radicals responsible for multiple damages to biomolecules, with consequent changes in their structure and functions.

The target cell concept $[5,6]$ states that tissue response to radiation exposure is governed by cell death in a target radiosensitive compartment, often the stem cell compartment, and that tissue regeneration depends on the surviving and proliferation of stem or progenitor cells. Thus in the case of intestinal mucosa, the severity of radiation damage and its regeneration capacity have long been paralleled to the level of cell death in the stem cell compartment. R-spondin1 , a potent intestinal stem cell growth factor and ligand of LGR5 receptor, protects mice from gastrointestinal syndrome $[7,8]$ and GLP-2, an intestinal growth factor, reduces both acute and late small intestinal damages following localized high-dose radiation exposure in the rat [9]. However, the increasing knowledge in radiation biology showed that the target cell concept does not reflect what is really happening in the vicinity of irradiated organs. All cell types are sensitive to ionizing radiations, and tissue scaring process initiates immediately after radiation exposure, involving all cell types and compartments of the tissue. Reduction of endothelial cells radiation-induced apottosis by basic fibroblast growth factor protects mice from gastrointestinal syndrome [10], and reduced small intestinal tissue damage following localized radiation exposure in PAI-1 -/- mice is associated with less endothelial cells apoptosis [11]. Tissue response to radiation exposure is considered as a continuum between very acute events and late tissue fibrosis. Conversely to normal scaring, tissue response to ionizing radiation can be considered as a chronic and self-maintained scaring process leading to fibrosis. The acute or prefibrotic phase is characterized by an inflammatory process with tissue damage essentially visible in the mucosal compartment. The young fibrosis shows immunocompetent cells accumulation and mesenchymal cells activation (fibroblasts and smooth muscle cells). Established fibrosis is paucicellular, with densification of scaring tissue and continuous matrix remodelling (Figure 1).

\section{Radiation-Induced Inflammation}

Ionizing radiations can be considered as a proinflammatory signal, and normal tissue response to radiation exposure is immediate and endures with time. Radiation-induced inflammatory response is initiated by the production of reactive oxygen/nitrate species, the induction of apoptosis and clonogenic cell death, mucosal breakdown, and the activation of the transcription of several proinflammatory cytokines, chemokines, and growth factors in the microvascular and mucosal compartments, presumably by recruited immune cells but also by enterocytes and residing cells, depending on the severity of tissue trauma $[12,13]$.

The vascular endothelium is a critical target compartment involved in tissue response to radiation exposure and strongly participates in the initiation and development of radiation lesions $[5,14]$. Irradiation of the vascular endothelium leads to endothelial cell apoptosis and the acquisition of a proinflammatory, prothrombotic, and antifibrinolytic phenotype, with increased secretion of soluble mediators such as cytokines, chemokines, and growth factors [15]. The increase in adhesion molecules expression such as VCAM1, ICAM-1, PECAM-1 as well as E and P selectins [16], and the expression of proinflammatory soluble mediators by irradiated endothelial cells activate resident macrophages and favour the early recruitment of polymorphonuclear neutrophils from the bloodstream. Neutrophils are recruited within minutes following tissue trauma, and their presence is characteristic of acute inflammation. Inflammatory process is then amplified by the recruitment and transmigration of monocytes and the activation of resident mast cells, both producing proinflammatory and profibrosing mediators such as IL-1 $\beta$, IL-6, IL- 8 , CXCL-1, CXCL-2, TNF- $\alpha$, or TGF$\beta$ [17-20]. The innate immune response carried out by 


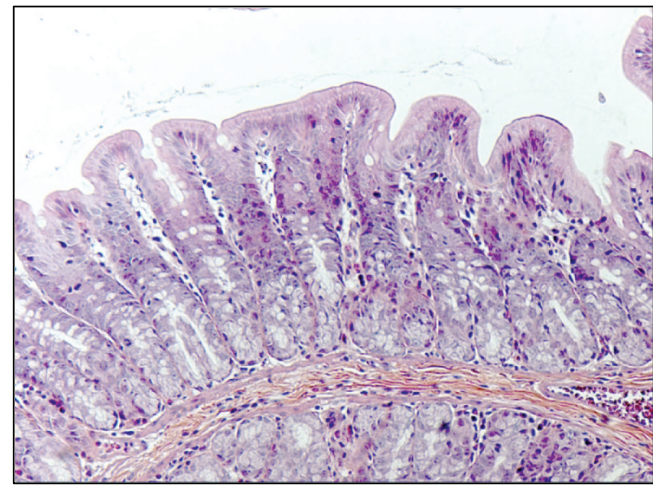

(a)

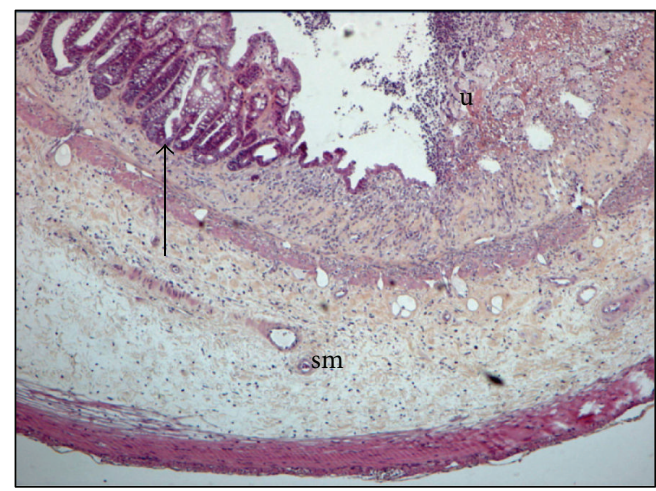

(b)

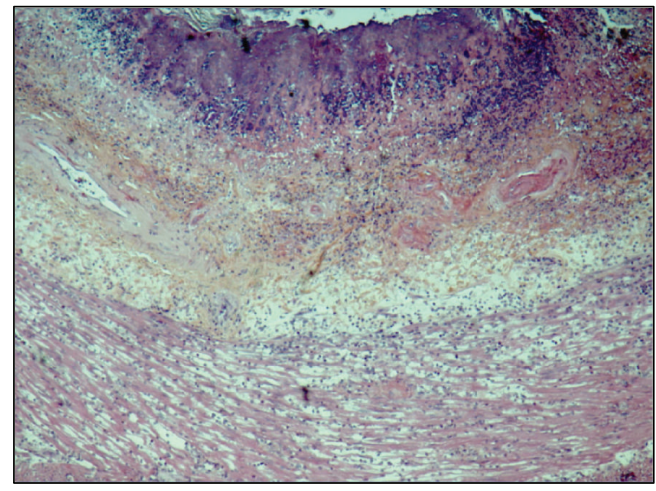

(c)

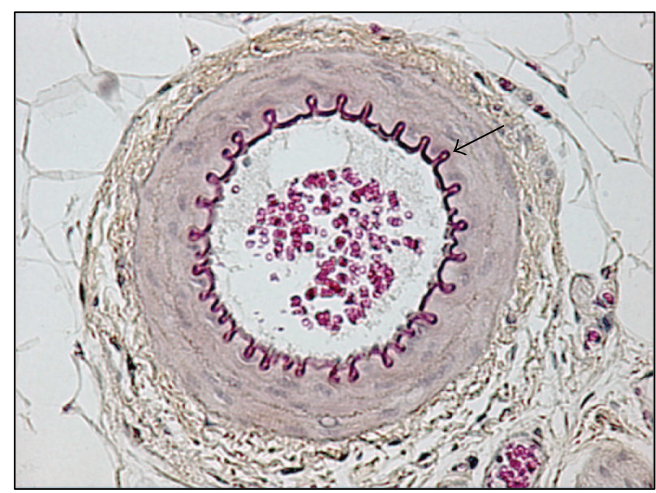

(d)

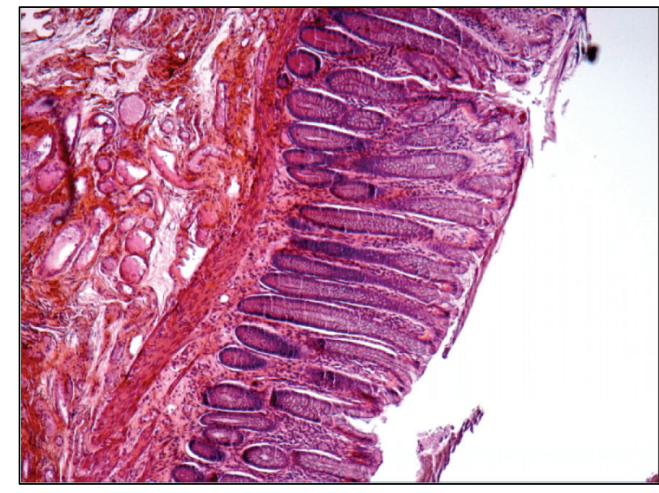

(f)

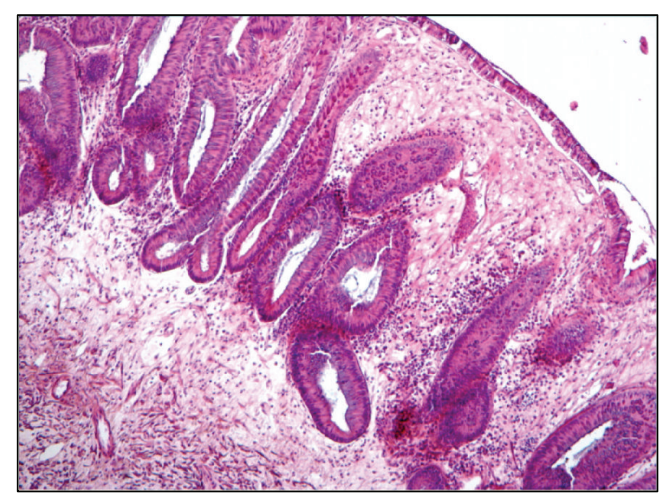

(g)

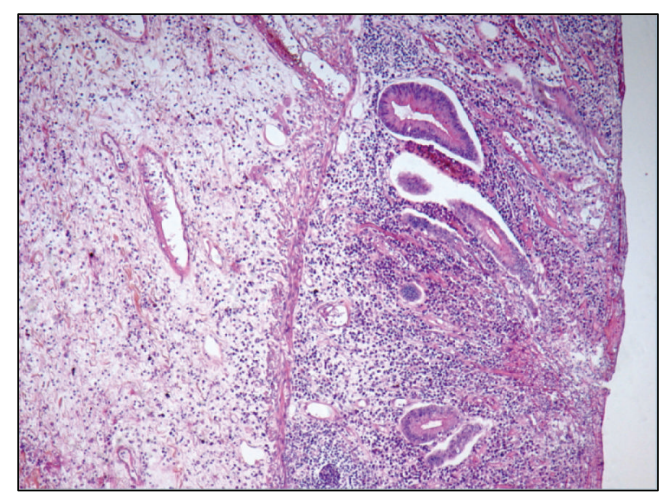

(h)

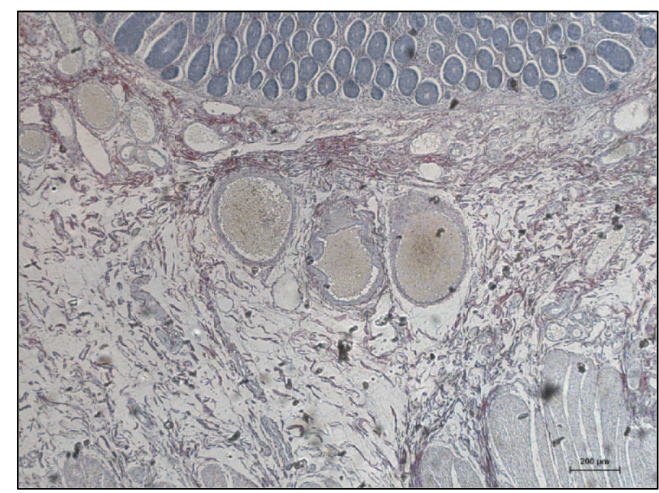

(i)

FIgure 1: Continued. 


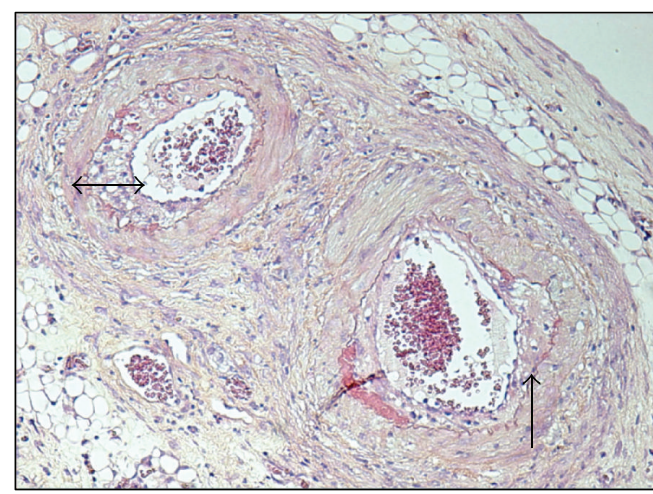

(e)

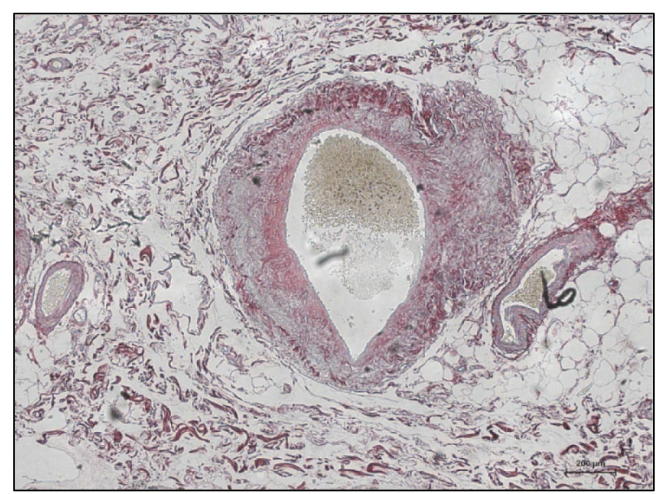

(j)

Figure 1: Left panel ((a) to (e)): Radiation-induced damage to the rectal wall following localized exposure to 27 Gy single dose in the rat. (a) Healthy mucosa. (b) Two weeks after exposure, tissue shows mucosal and submucosal inflammation, with mucosal ulceration (u) and submucosal (sm) oedema. Glandular recovering (arrow) alternates with ulcerated areas. (c) Height weeks after exposure, underlying the ulcerated areas, the entire rectal wall is pathologic. The mucosa and submucosa show still severe inflammation, with tissue necrosis at the luminal frontier (purple coloration). Inflammation reaches the oedematous and dystrophic external muscular layers. Note that the muscularis mucosa disappeared. (e) Eight weeks after exposure, severe epithelial, submucosal, and muscular damage is associated with dystrophic submucosal and mesenteric vessels. The elastic layer is dystrophic (arrow) compared to the healthy vessels ((d), arrow) and, neointimal hyperplasia reduces the vessel lumen (double arrow). HeS staining, original magnification $\times 100((\mathrm{a}),(\mathrm{d}),(\mathrm{e}))$ or $\times 40((\mathrm{~b})$, (c)), pictures Agnès Francois. Right panel radiation-induced damage to the rectal wall in patients treated for rectal adenocarcinoma, 5 to 7 weeks following the end of radiation therapy ( $45 \mathrm{~Gy}$ ). (f) Healthy rectal mucosa. (g): Epithelial atypia with mucosal oedema and inflammation. Crypt positioning is disorganized and some are bifid and show hyperplasia, both signing epithelial regeneration. (h) Severe mucosal ulceration with submucosal oedema and dense inflammatory infiltrate. Crypt number is drastically reduced. (j) Dystrophic submucosal arteriole, with collagen deposition in the vessel wall and neointimal hyperplasia reducing the vascular lumen. (f), (g), (h) HeS staining, (i), (j) Sirius Red staining. Original magnification $\times 40$, pictures Agnès François.

macrophages, neutrophils, and mast cells is supported by the adaptative immune response assumed by the $\mathrm{B}$ and $\mathrm{T}$ lymphocytes (Figure 2).

4.1. Innate or Unspecific Immune Response. Innate immunity insures organisms against pathogens. The first line of defence is the physical barrier carried out by the intestinal mucosa, in which multiple immune cells (macrophages, polymorphonuclear neutrophils, mast cells reside, and innate lymphoid cells) implicated in the immediate unspecific reactions to antigens shared by a plethora of pathogens.

4.1.1. Macrophages. Resident macrophages and circulating monocytes are the sensors of tissue homeostasis breakdown. Upon tissue injury, activated macrophages release a cytokine and chemokine "soup" to recruit neutrophils [21]. Once in the injured tissue, neutrophils can in turn emit signals to favour monocyte recruitment from the bloodstream. Following extravasation, monocytes differentiate into dendritic cells, resident macrophages (M2 type), or inflammatory macrophages (M1 type) depending on the tissue context [22]. Macrophages play a crucial role in the development of inflammation but also in its resolution and tissue regeneration, notably in clearing out tissues from apoptotic neutrophils, bacteria, and cells and tissue debris. The termination of inflammation is orchestrated by many factors. For example, neutrophil apoptosis can trigger several feed-back signals which dampen further neutrophil recruitment, and neutrophil phagocytosis by macrophages can induce in the latters ones a switch in the nature of secreted mediators toward a suppression of the inflammatory response (decreased TNFalpha and increased TGF-beta and IL-10 secretions) [21].

Fibrotic small bowel of patients with radiation enteritis show altered expression of many genes implicated in stress response, inflammation, and antioxidant metabolism, among them is increased expression of MIP-2, a member of C-X-C chemokine family secreted by monocytes and macrophages and chemoattractive for neutrophils [23]. Rectal biopsies from 33 patients treated with radiotherapy for nongastrointestinal pelvic carcinoma show increased density of macrophages at the end of the second week of treatment mainly in the mucosal compartment [24]. In 17 patients treated for prostate carcinoma, rectal biopsies show increased macrophage invasion 2 and 6 weeks after the commencement of radiotherapeutic treatment [25]. Macrophage immunostaining is increased in the rat small intestine 2 weeks after localized exposure to single dose (21 Gy) or fractionated dose of X-radiation $(8 \times 5.6 \mathrm{~Gy})$ [26]. Finally, radiation proctitis in mice 2 and 14 weeks following 27 Gy single-dose radiation exposure is associated with increased macrophage density in all tissue compartments [20].

Broadly, authors often report macrophage invasion in irradiated intestine in human tissues as well as in preclinical animal models, but no data exist concerning the putative role played by macrophages in the initiation and development of gut radiation damage. Macrophages, but also neutrophils, are followed in radiation lesions to establish radiation injury scores and put in evidence eventual beneficial effects associated with therapeutic strategies. The understanding of the roles played by macrophages in radiation damage may 


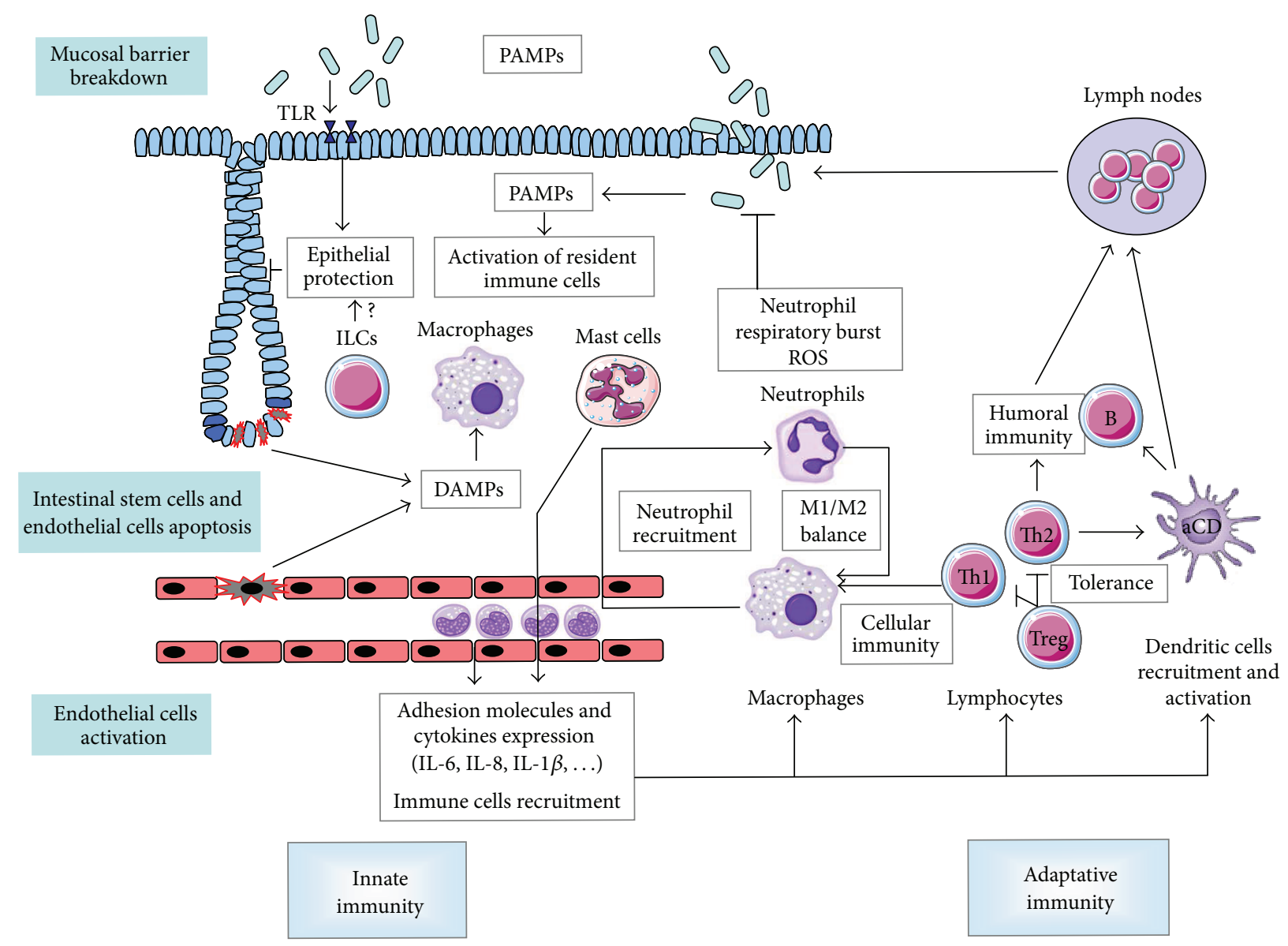

FIGURE 2: Radiation exposure to the gut mucosa induces epithelial stem cells apoptosis and clonogenic cell death, endothelial cells death and activation, and mucosal barrier breakdown. Innate immunity: resident immune cells such as macrophages and mast cells are activated by DAMPs generated by dead cells and PAMPs coming from mucosal breakdown. Activated endothelial cells express adhesion molecules and cytokines favouring immune cells recruitment into the injured tissue. Activated macrophages increase neutrophil recruitment, which in turn emit signals favouring monocytes recruitment from the blood stream. The radiation-induced tissue M1/M2 balance is unknown. Adaptative immunity: Th1 lymphocytes can activate innate immune cells and favour cell-mediated immunity, whereas Th2 favour humoral immunity via B cells. The balance in irradiated gut tissue is in favour of a Th2 orientation. Treg maintain immune tolerance. Resident and recruited dendritic cells are activated and carry out the link with lymph nodes and the establishment of specific immune response. PAMPs are also detected by TLRs, whose activation can protect epithelium against radiation damage. ILCs play a role in epithelial homeostasis, but their role after radiation exposure is unknown.

help to find new therapeutic options. For instance, to favour macrophage M2 phenotype or neutrophil apoptosis may help macrophages to dampen inflammation and would be an interesting therapeutic option but necessitate a refined knowledge of the roles of the different cell types in the successive phases of intestinal tissue response to radiation exposure.

4.1.2. Polymorphonuclear Neutrophils. Neutrophils, with their phagocytic and microbicidal capacities, play a key role in the protection of organisms against infections and participate to the inflammatory response of injured tissues. Radiation-induced intestinal mucosal and vascular barrier breakdown leads to bacterial translocation and to immediate recruitment of neutrophils to the site of injury. The microbicidal properties of the reactive oxygen species (ROS) produced by the so-called neutrophils "respiratory burst" [27] are crucial in the management of the first steps of gastrointestinal infections and inflammation. However, excessive and sustained ROS production may damage healthy cells and tissues and participate in the progression and the chronicity of radiation injury to the intestinal wall. Radiation-damaged tissues, included during the late fibroatrophic phase, often present sustained oxidative stress [28], and the protective effects of probiotics in the pathogenesis of radiation injury to the digestive tract may in part rely on their antioxidant properties [29]. Neutrophil influx is observed in the rat small intestine until 26 weeks after exposure to localized single (18, 21, 29.6 Gy) or fractionated $(16 \times 4.2 \mathrm{~Gy}) \mathrm{X}$-radiation [30, 31]. In mice, radiation proctitis following $27 \mathrm{~Gy}$ single-dose exposure shows increased neutrophil numbers in the inflamed and 
fibrosed areas 2 and 14 weeks postirradiation. The expression of CXCL-1 and CXCL-2, both having a strong neutrophil chemoattractant activity, is increased in irradiated tissues as soon as 3 hours postirradiation, [20]. In humans, biopsies from rectal mucosa of patients at 2 and 6 weeks during the course of ongoing radiotherapy for prostate carcinoma show increased neutrophil numbers [25].

The precise roles of neutrophils in radiation enteritis and proctitis are still unknown and remain ambiguous. Neutrophils are generally considered deleterious in intestinal inflammation. However, in radiation-induced proctitis in mice, increased neutrophil chemoattractant expression and subsequently higher neutrophil numbers were associated with less tissue damage in mast cells-deficient mice [20]. Several studies have reported a potential role of acute neutrophil influx in tissue protection. In Smad3 knockout mice receiving $30 \mathrm{~Gy}$ on the flank skin, reduced tissue radiation damage is associated with increased acute neutrophil influx [32]. In a model of septic shock following cecal ligation and puncture in mice, Alves-Filho et al. noticed that IL33 favours neutrophils chemotaxis to the site of infection and reduces animal mortality. IL-33-treated animals exhibit enhanced neutrophil influx in the peritoneal cavity and better bacterial clearance [33]. Neutrophil infiltration is a hallmark of irradiated tissues, and several studies are necessary to discriminate between their useful and detrimental properties in the initiation and progression of radiation damage to the digestive tract.

4.1.3. Mast Cells. Mast cells are resident immune cells mainly located in tissues in close contact with the external milieu (gut, lung, and skin) thus participating in innate and adaptative immune reactions $[34,35]$. Mast cells are able to secrete a huge number of proinflammatory, profibrosing, vasoactive, and mitogenic soluble mediators (stocked in their granules or newly synthesized) in response to various physiological and pathological situations. Mast cells have been known for a long time to be implicated in allergic reactions but are also involved in several inflammatory and fibrotic disorders [36]. Numerous studies remain necessary to define the precise roles of mast cells in the initiation and development of radiation damage to the intestine. In particular, few data exist, and the diversity of irradiation models generates controversy about mast cells response to irradiation in vitro and in vivo. Globally, in vitro, mast cells are rather radioresistant compared to other immune cells [37]. In vivo, mast cells also appear quite radioresistant in mice following $20 \mathrm{~Gy}$ fractionated total body irradiation, with no evidence of radiationinduced degranulation [38]. Conversely in the rat, intestinal mast cells are severely depleted after $3.5,5$, or $10 \mathrm{~Gy}$ singledose total body radiation exposure $[39,40]$. Human proctitis following radiation therapy for rectal adenocarcinoma is associated with mast cell hyperplasia in the mucosa and submucosa, in muscularis propria, in the dystrophic vessel wall, and more globally in the areas of inflammation and collagen deposition [20]. Mast-cells-deficient rats develop severe acute mucosal damage compared to wild-type animals following localized radiation exposure of the small intestine to $21 \mathrm{~Gy}$-single dose but are protected from late tissue fibrosis [41]. Despite reduced collagen accumulation in fibrotic lesions of mast-cells-deficient rats, TGF $\beta-1$ mRNA expression in irradiated tissues is similar to their control littermates. Authors suggest that mast cells participate to the profibrosing effect of TGF $\beta-1$. Experimental radiation proctitis in mice shows mast cells hyperplasia, and mast-cellsdeficient mice are protected from both acute ( 2 weeks) and chronic (14 weeks) rectal damage [20], suggesting again that mast cells play a role in the radiation-induced inflammatory and fibrosing processes in the gut.

Besides a wide range of proinflammatory and profibrosing mediators, mast cells also secrete chymase and tryptase, two proteases implicated in numerous biological processes. Mast cell chymase is involved in vascular, inflammatory, and fibrosing diseases. Chymase can activate matrix metalloproteases, degrades matrix proteins such as fibronectin and vitronectin, and potentiates the biological actions of IL$1 \beta$, TGF $\beta-1$, endothelin-1, and angiotensin II [42]. Mast cell tryptase is involved in the regulation of vascular permeability, proliferation, and contraction of smooth muscle cells, angiogenesis, inflammation and fibrosis [43]. Mast cell tryptase can also activate protease-activated receptor-2 (PAR-2). PARs are G-protein-coupled receptors expressed on the surface of many cell types including enterocytes, smooth muscle cells, immune cells, endothelial cells, and stromal cells. PAR-2 is implicated in various biological functions such as intestinal ion transport, motility, vascular tone, inflammation, and cell migration and proliferation in response to various traumas [44]. PAR-2 expression and activation are increased after radiation exposure of the small intestine of the wild-type rat, with significant attenuation in mast cells-deficient rats, showing that deleterious effects of mast cells during the fibroproliferative phase of intestinal radiation injury may partly occur via PAR-2 activation $[45,46]$. In vitro, exogenously added mast cell chymase or tryptase can turn human colonic muscularis propria smooth muscle cells toward a migrating/proliferating and proinflammatory phenotype, making mast cells putative participants in the in vivo radiationinduced inflammatory process and dystrophy of the muscularis propria [20]. Finally, in vitro exposure of endothelial cells to mast-cells-conditioned medium exacerbates the radiationinduced overexpression of inflammatory genes such as IL-6, IL-8, CXCL-2, and E-selectin [47], suggesting that mast cells may participate in the activation of vascular endothelium and in the global tissue inflammation following radiation exposure. There is still a lot to be done to understand the roles of mast cells in the response of healthy intestinal tissue to radiation exposure, during the initiation/inflammatory phase of radiation injury and during the recovery process, especially when chronicity takes hold.

4.1.4. Innate Lymphoid Cells (Ilcs). ILCs regroup various lymphoid cells populations, among which NK cells implicated in the maintenance of epithelial homeostasis and integrity. They produce numerous cytokines and growth factors as potent stimulators of epithelial cell growth [48]. For example, IL-22, expressed by Th cells and ILCs and with a receptor located on stromal mesenchymal and epithelial cells, triggers the production of genes involved in epithelial cell 
differentiation and survival and has been shown to protect intestinal Paneth and stem cells against immune-mediated tissue damage in a model of graft-versus-host disease in mice. Reciprocally, deficiency in IL-22 led to loss of ILCs and subsequent intestinal barrier disruption and increased tissue damage, showing mutual interactions between immune and epithelial compartments [49]. To our knowledge, no data exist concerning gut ILCs in the development of radiation gut damage following localized exposure.

4.1.5. Pattern Recognition Receptors (PRRs). Innate response relies on the activation of receptors recognizing pathogens molecular motifs (for Pathogen-Associated Molecular Patterns (PAMPS)) and referred to as Pattern Recognition Receptors (PRRs). Among the different PRRs, Toll-Like Receptors (TLRs) are able to recognize Damage-Associated Molecular Patterns (DAMPs) produced by injured tissues and damaged and activated cells, thus playing a putative role in tissue homeostasis and repair such as postradiation exposure [50]. TLRs are expressed on the surface of multiple cell types such as immune cells, fibroblasts, or intestinal epithelial cells. Pretreatment with the TLR5 receptor ligand flagellin is protective against sublethal doses of whole body irradiation in mice and primates [51] and protects gut mucosal tissue from apoptosis following $8 \mathrm{~Gy}$ total body irradiation in mice [52]. In the same way, probiotics inhibit TLR4/NFkB signalling and reduce the severity of experimental colitis [53]. Probiotics also reduce radiation-induced epithelial injury and improve crypt survival in mice following $12 \mathrm{~Gy}$ whole body irradiation in a TLR-2/COX-2-dependent manner [54]. There are still no data on the role of TLRs signalling and the development of radiation enteritis and proctitis following high-dose localized intestinal radiation exposure. Working in this direction would warranty interesting breakthroughs and probably open new therapeutic windows to prevent or mitigate severe gut radiation injury.

4.2. Adaptative Immune Response. The adaptative immunity consists of immune cell response to specific antigens. This specific response necessitates the presentation to $\mathrm{B}$ and $\mathrm{T}$ lymphocytes of antigens coming from pathogens by specialized presenting cells such as dendritic cells. The rapid innate response following tissue radiation exposure changes the composition of the microenvironment and favours dendritic cells maturation, antigens presentation and the induction of clonal proliferation of selected immune cells. Adaptative immunity amplifies the first line innate response, so innate and adaptative immunities complement to each other.

Lymphocyte infiltrate is a common feature of irradiated normal tissues, especially in the subacute and chronic inflammatory phases of radiation damage. Following antigen presentation, naïve $\mathrm{CD} 4^{+} \mathrm{T}$ cells can differentiate into different T cells subsets such as Th1, Th2, Th17, or Treg, each showing specific cytokine expression profiles controlled by distinct transcription factors, and assuming various redundant or opposed roles in the tissue immune response to injury.

Immune-modulation properties of ionizing radiation are used in the context of antitumor radiation therapy [55].
In normal tissues, even if lymphocytes subsets have different radiation sensitivities, radiation exposure in total body configuration is used for its immune-suppressive properties. The effects of localized irradiation on lymphocytes are more complex and depend on the irradiated organ, the size of the irradiation field, and the delivery protocol (fraction schedule and total administered dose). $\mathrm{CD} 4^{+} \mathrm{T}$ cells are crucial in the adaptative immune response as they help Bcell to produce antibodies, activate $\mathrm{CD} 8^{+}$cytotoxic $\mathrm{T}$ cells, and are implicated in the recruitment of innate immune cells such as neutrophils and monocytes/macrophages to the site of tissue damage. $\mathrm{CD}^{+} \mathrm{T}$ cells polarisation into Th1, Th2, Th17, and Treg subsets is acquired depending on the tissue cytokine context and is revealed by the activation of specific transcription factors, the expression of several chemokine receptors, a specific cytokine expression profile for each subset, and the activation of preferential partner cells triggering specific immunity [56]. Briefly, Thl cells are responsible for cell-mediated immunity, stimulate macrophages, and thus contribute to the elimination of intracellular pathogens and promote tumoricidal activity. The Thl profile is mainly associated with autoimmune diseases. Th2 cells mainly stimulate humoral immunity with B-cell activation and are implicated in allergic reactions. Th17 cells recruit neutrophils and are implicated in the elimination of extracellular pathogens and also participate in the development of autoimmune diseases. Treg cells ensure immunotolerance. Globally, radiation exposure, by its direct effects on adaptative immune cells and by the generation of innate immune response and inflammation, triggers an imbalance in immune populations which still remains obscure [57]. For example, abdominal irradiation in the rat increases Treg populations in the intestine with impaired ability to control effectors $\mathrm{T}$ cells that may imbalance immune tolerance, favour inflammation, and compromise normal tissue repair [58]. Still in the rat, fractionated irradiation localized on the colorectum modifies the $\mathrm{CD} 4^{+} \mathrm{T}$ helper polarization through a Th2 profile. The imbalance in favour of Th2 cells persists until 6 months postirradiation [59]. Th1/Th2 imbalance has been shown to play a determining role in human inflammatory bowel disease, with the development of a Th1 profile in Crohn's disease and a Th2 profile in ulcerative colitis. Continuing immune imbalance may play a significant role in the chronicity of radiation lesions, and further studies are necessary to describe precisely the nature of infiltrating immune cells, to understand the modalities of their recruitment, and finally to highlight the roles played by adaptative immunity in the context of normal digestive tissue response to radiation exposure.

In conclusion, normal gut tissue response to radiation exposure is the result of cell death and activation in all tissue compartments, with a strong oxidative and immunoinflammatory component. The precise roles of the different resident and recruited immune cells described in irradiated normal tissues are still obscure, as well as the part played by innate and adaptative immunities. Strong evidence suggests that ongoing researches in this direction warranty opportunities to discover new therapeutic tools to manage normal tissue radiation damage. Given the relatively poor therapeutic efficiency of "classic" anti-inflammatory strategies, it appears 
necessary to increase the knowledge concerning enduring oxidative stress, vascular endothelial cell activation, immune cells recruitment and their phenotypic orientations such as M1/M2 macrophages and lymphocytes Th1/Th2/Th17/Treg balances, and finally the conditions necessary to the resolution of radiation-induced inflammation. This may help the understanding of the benefit/risk ratio of the radiationinduced immunoinflammatory response and offer considerable improvement in the benefit of anticancer radiotherapy as well as in the management of normal tissues side effects.

\section{References}

[1] J. W. Denham and M. Hauer-Jensen, "The radiotherapeutic injury-a complex "wound"', Radiotherapy and Oncology, vol. 63, no. 2, pp. 129-145, 2002.

[2] H. J. N. Andreyev, A. Wotherspoon, J. W. Denham, and M. Hauer-Jensen, "'Pelvic radiation disease": new understanding and new solutions for a new disease in the era of cancer survivorship," Scandinavian Journal of Gastroenterology, vol. 46, no. 4, pp. 389-397, 2011.

[3] H. J. N. Andreyev, A. Wotherspoon, J. W. Denham, and M. Hauer-Jensen, "Defining pelvic-radiation disease for the survivorship era," The Lancet Oncology, vol. 11, no. 4, pp. 310-312, 2010.

[4] M. Bjerknes and H. Cheng, "Clonal analysis of mouse intestinal epithelial progenitors," Gastroenterology, vol. 116, no. 1, pp. 7-14, 1999.

[5] S. M. Bentzen, "Preventing or reducing late side effects of radiation therapy: radiobiology meets molecular pathology," Nature Reviews Cancer, vol. 6, no. 9, pp. 702-713, 2006.

[6] F. A. Stewart and W. Dörr, "Milestones in normal tissue radiation biology over the past 50 years: from clonogenic cell survival to cytokine networks and back to stem cell recovery," International Journal of Radiation Biology, vol. 85, no. 7, pp. 574586, 2009.

[7] P. Bhanja, S. Saha, R. Kabarriti et al., "Protective role of R-spondin1, an intestinal stem cell growth factor, against radiationinduced gastrointestinal syndrome in mice," PLoS ONE, vol. 4, no. 11, Article ID e8014, 2009.

[8] W. de Lau, N. Barker, T. Y. Low et al., "Lgr5 homologues associate with Wnt receptors and mediate R-spondin signalling," Nature, vol. 476, no. 7360, pp. 293-297, 2011.

[9] S. Torres, L. Thim, F. Milliat et al., "Glucagon-like peptide-2 improves both acute and late experimental radiation enteritis in the rat," International Journal of Radiation Oncology Biology Physics, vol. 69, no. 5, pp. 1563-1571, 2007.

[10] F. Paris, Z. Fuks, A. Kang et al., "Endothelial apoptosis as the primary lesion initiating intestinal radiation damage in mice," Science, vol. 293, no. 5528, pp. 293-297, 2001.

[11] R. Abderrahmani, A. François, V. Buard et al., "Effects of pharmacological inhibition and genetic deficiency of plasminogen activator inhibitor-1 in radiation-induced intestinal injury," International Journal of Radiation Oncology Biology Physics, vol. 74, no. 3, pp. 942-948, 2009.

[12] A. Polistena, L. B. Johnson, S. Ohiami-Masseron et al., "Local radiotherapy of exposed murine small bowel: apoptosis and inflammation," BMC Surgery, vol. 8, article 1, 2008.
[13] Z. Y. Ong, R. J. Gibson, J. M. Bowen et al., "Pro-inflammatory cytokines play a key role in the development of radiotherapyinduced gastrointestinal mucositis," Radiation Oncology, vol. 5, no. 1, article 22, 2010.

[14] F. Milliat, A. François, R. Tamarat, and M. Benderitter, "Role of endothelium in radiation-induced normal tissue damages," Annales de Cardiologie et d'Angeiologie, vol. 57, no. 3, pp. 139$148,2008$.

[15] J. Wang, M. Boerma, Q. Fu, and M. Hauer-Jensen, "Significance of endothelial dysfunction in the pathogenesis of early and delayed radiation enteropathy," World Journal of Gastroenterology, vol. 13, no. 22, pp. 3047-3055, 2007.

[16] M. Mollà, M. Gironella, R. Miquel et al., "Relative roles of ICAM-1 and VCAM-1 in the pathogenesis of experimental radiation-induced intestinal inflammation," International Journal of Radiation Oncology Biology Physics, vol. 57, no. 1, pp. 264273, 2003.

[17] M. Berthrong and L. F. Fajardo, "Radiation injury in surgical pathology. Part II. Alimentary tract," American Journal of Surgical Pathology, vol. 5, no. 2, pp. 153-178, 1981.

[18] C. W. Langberg, "Expression of fibrogenic cytokines in rat small intestine after fractionated irradiation," Radiotherapy and Oncology, vol. 32, no. 1, pp. 29-36, 1994.

[19] K. K. Richter, C. W. Langberg, C. C. Sung, and M. Hauer-Jensen, "Increased transforming growth factor $\beta$ (TGF- $\beta$ ) immunoreactivity is independently associated with chronic injury in both consequential and primary radiation enteropathy," International Journal of Radiation Oncology Biology Physics, vol. 39, no. 1, pp. 187-195, 1997.

[20] K. Blirando, F. Milliat, I. Martelly, J. C. Sabourin, M. Benderitter, and A. François, "Mast cells are an essential component of human radiation proctitis and contribute to experimental colorectal damage in mice," American Journal of Pathology, vol. 178, no. 2, pp. 640-651, 2011.

[21] O. Soehnlein and L. Lindbom, "Phagocyte partnership during the onset and resolution of inflammation," Nature Reviews Immunology, vol. 10, no. 6, pp. 427-439, 2010.

[22] S. Gordon, “The macrophage: past, present and future," European Journal of Immunology, vol. 37, no. 1, pp. S9-S17, 2007.

[23] M. C. Vozenin-Brotons, F. Milliat, C. Linard et al., "Gene expression profile in human late radiation enteritis obtained by highdensity cDNA array hybridization," Radiation Research, vol. 161, no. 3, pp. 299-311, 2004.

[24] N. Hovdenak, L. F. Fajardo, and M. Hauer-Jensen, "Acute radiation proctitis: a sequential clinicopathologic study during pelvic radiotherapy," International Journal of Radiation Oncology Biology Physics, vol. 48, no. 4, pp. 1111-1117, 2000.

[25] N. Hovdenak, J. Wang, C. C. Sung, T. Kelly, L. F. Fajardo, and M. Hauer-Jensen, "Clinical significance of increased gelatinolytic activity in the rectal mucosa during external beam radiation therapy of prostate cancer," International Journal of Radiation Oncology Biology Physics, vol. 53, no. 4, pp. 919-927, 2002.

[26] M. Boerma, J. Wang, K. K. Richter, and M. Hauer-Jensen, "Orazipone, a locally acting immunomodulator, ameliorates intestinal radiation injury: a preclinical study in a novel rat model," International Journal of Radiation Oncology Biology Physics, vol. 66, no. 2, pp. 552-559, 2006.

[27] M. Ciz, P. Denev, M. Kratchanova, O. Vasicek, G. Ambrozova, and A. Lojek, "Flavonoids inhibit the respiratory burst of neutrophils in mammals," Oxidative Medicine and Cellular Longevity, vol. 2012, Article ID 181295, 6 pages, 2012. 
[28] M. E. C. Robbins and W. Zhao, "Chronic oxidative stress and radiation-induced late normal tissue injury: a review," International Journal of Radiation Biology, vol. 80, no. 4, pp. 251259, 2004.

[29] B. G. Spyropoulos, E. P. Misiakos, C. Fotiadis, and C. N. Stoidis, "Antioxidant properties of probiotics and their protective effects in the pathogenesis of radiation-induced enteritis and colitis," Digestive Diseases and Sciences, vol. 56, no. 2, pp. 285-294, 2011.

[30] A. François, F. Milliat, M. C. Vozenin-Brotons, D. Mathe, and N. M. Griffiths, "'In-field" and "out-of-field" functional impairment during subacute and chronic phases of experimental radiation enteropathy in the rat," International Journal of Radiation Biology, vol. 79, no. 6, pp. 437-449, 2003.

[31] J. Wang, H. Zheng, X. Ou, L. M. Fink, and M. HauerJensen, "Deficiency of microvascular thrombomodulin and upregulation of protease-activated receptor-1 in irradiated rat intestine: possible link between endothelial dysfunction and chronic radiation fibrosis," American Journal of Pathology, vol. 160, no. 6, pp. 2063-2072, 2002.

[32] K. C. Flanders, B. M. Ho, P. R. Arany et al., "Absence of Smad3 induces neutrophil migration after cutaneous irradiation: possible contribution to subsequent radioprotection," American Journal of Pathology, vol. 173, no. 1, pp. 68-76, 2008.

[33] J. C. Alves-Filho, F. Snego, F. O. Souto et al., "Interleukin-33 attenuates sepsis by enhancing neutrophil influx to the site of infection," Nature Medicine, vol. 16, no. 6, pp. 708-712, 2010.

[34] D. D. Metcalfe, D. Baram, and Y. A. Mekori, "Mast cells," Physiological Reviews, vol. 77, no. 4, pp. 1033-1079, 1997.

[35] S. Tete, D. Tripodi, M. Rosati et al., "Role of mast cells in innate and adaptive immunity," Journal of Biological Regulators \& Homeostatic Agents, vol. 26, no. 2, pp. 193-201, 2012.

[36] M. A. Beaven, "Our perception of the mast cell from Paul Ehrlich to now," European Journal of Immunology, vol. 39, no. 1, pp. 11-25, 2009.

[37] F. Nakayama, K. Müller, A. Hagiwara, R. Ridi, M. Akashi, and V. Meineke, "Involvement of intracellular expression of FGF12 in radiation-induced apoptosis in mast cells," Journal of Radiation Research, vol. 49, no. 5, pp. 491-501, 2008.

[38] B. P. Soule, J. M. Brown, N. M. Kushnir-Sukhov, N. L. Simone, J. B. Mitchell, and D. D. Metcalfe, "Effects of gamma radiation on FceRI and TLR-mediated mast cell activation," Journal of Immunology, vol. 179, no. 5, pp. 3276-3286, 2007.

[39] D. M. Sedgwick and A. Ferguson, "Dose-response studies of depletion and repopulation of rat intestinal mucosal mast cells after irradiation," International Journal of Radiation Biology, vol. 65, no. 4, pp. 483-495, 1994.

[40] A. François, J. Aigueperse, P. Gourmelon, W. K. Macnaughton, and N. M. Griffiths, "Exposure to ionizing radiation modifies neurally-evoked electrolyte transport and some inflammatory responses in rat colon in vitro," International Journal of Radiation Biology, vol. 73, no. 1, pp. 93-101, 1998.

[41] H. Zheng, J. Wang, and M. Hauer-Jensen, "Role of mast cells in early and delayed radiation injury in rat intestine," Radiation Research, vol. 153, no. 5, pp. 533-539, 2000.

[42] H. Saito, "Role of mast cell proteases in tissue remodeling," Chemical Immunology and Allergy, vol. 87, pp. 80-84, 2005.

[43] J. Hallgren and G. Pejler, "Biology of mast cell tryptase: an inflammatory mediator," The FEBS Journal, vol. 273, no. 9, pp. 1871-1895, 2006.

[44] S. R. Macfarlane, M. J. Seatter, T. Kanke, G. D. Hunter, and R. Plevin, "Proteinase-activated receptors," Pharmacological Reviews, vol. 53, no. 2, pp. 245-282, 2001.
[45] J. Wang, H. Zheng, M. D. Hollenberg, S. J. Wijesuriya, X. $\mathrm{Ou}$, and M. Hauer-Jensen, "Up-regulation and activation of proteinase-activated receptor 2 in early and delayed radiation injury in the rat intestine: influence of biological activators of proteinase-activated receptor 2," Radiation Research, vol. 160, no. 5, pp. 524-535, 2003.

[46] J. Wang and M. Hauer-Jensen, "Neuroimmune interactions: potential target for mitigating or treating intestinal radiation injury," British Journal of Radiology, vol. 80, no. 1, pp. S41-S48, 2007.

[47] K. Blirando, M. Hneino, I. Martelly, M. Benderitter, F. Milliat, and A. François, "Mast cells and ionizing radiation induce a synergistic expression of inflammatory genes in endothelial cells by a mechanism involving p38 $\alpha$ MAP kinase and (p65) NF$\kappa \mathrm{B}$ activation," Radiation Research, vol. 178, no. 6, pp. 556-567, 2012.

[48] H. Spits and J. P. Di Santo, "The expanding family of innate lymphoid cells: regulators and effectors of immunity and tissue remodeling," Nature Immunology, vol. 12, no. 1, pp. 21-27, 2011.

[49] A. M. Hanash, J. A. Dudakov, G. Hua et al., "Interleukin-22 protects intestinal stem cells from immune-mediated tissue damage and regulates sensitivity to graft versus host disease," Immunity, vol. 37, no. 2, pp. 339-350, 2012.

[50] D. Schaue and W. H. McBride, "Links between innate immunity and normal tissue radiobiology," Radiation Research, vol. 173, no. 4, pp. 406-417, 2010.

[51] L. G. Burdelya, V. I. Krivokrysenko, T. C. Tallant et al., "An agonist of toll-like receptor 5 has radioprotective activity in mouse and primate models," Science, vol. 320, no. 5873, pp. 226230, 2008.

[52] R. M. Jones, J. W. Mercante, and A. S. Neish, "Reactive oxygen production induced by the gut microbiota: pharmacotherapeutic implications," Current Medicinal Chemistry, vol. 19, no. 10, pp. 1519-1529, 2012.

[53] S. K. Lee, H. J. Kim, and S. G. Chi, "Saccharomyces boulardii reduced intestinal inflammation in mice model of 2,4,6trinitrobencene sulfonic acid induced colitis: based on microarray," The Korean Journal of Gastroenterology, vol. 55, no. 1, pp. 33-45, 2010.

[54] M. A. Ciorba, T. E. Riehl, M. S. Rao et al., "Lactobacillus probiotic protects intestinal epithelium from radiation injury in a TLR-2/cyclo-oxygenase-2-dependent manner," Gut, vol. 61, no. 6, pp. 829-838, 2012.

[55] E. L. Kachikwu, K. S. Iwamoto, Y. P. Liao et al., "Radiation enhances regulatory T cell representation," International Journal of Radiation Oncology, Biology, Physics, vol. 81, no. 4, pp. 11281135, 2011.

[56] J. Zhu and W. E. Paul, "Heterogeneity and plasticity of T helper cells," Cell Research, vol. 20, no. 1, pp. 4-12, 2010.

[57] D. Schaue and W. H. McBride, "T lymphocytes and normal tissue responses to radiation," Frontiers in Oncology, vol. 2, p. $119,2012$.

[58] F. Billiard, V. Buard, M. Benderitter, and C. Linard, "Abdominal $\gamma$-radiation induces an accumulation of function-impaired regulatory T cells in the small intestine," International Journal of Radiation Oncology Biology Physics, vol. 80, no. 3, pp. 869876, 2011.

[59] O. Grémy, M. Benderitter, and C. Linard, "Acute and persisting Th2-like immune response after fractionated colorectal $\gamma$ irradiation," World Journal of Gastroenterology, vol. 14, no. 46, pp. 7075-7085, 2008. 


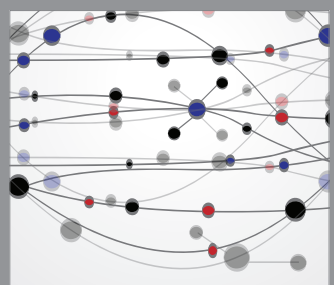

The Scientific World Journal
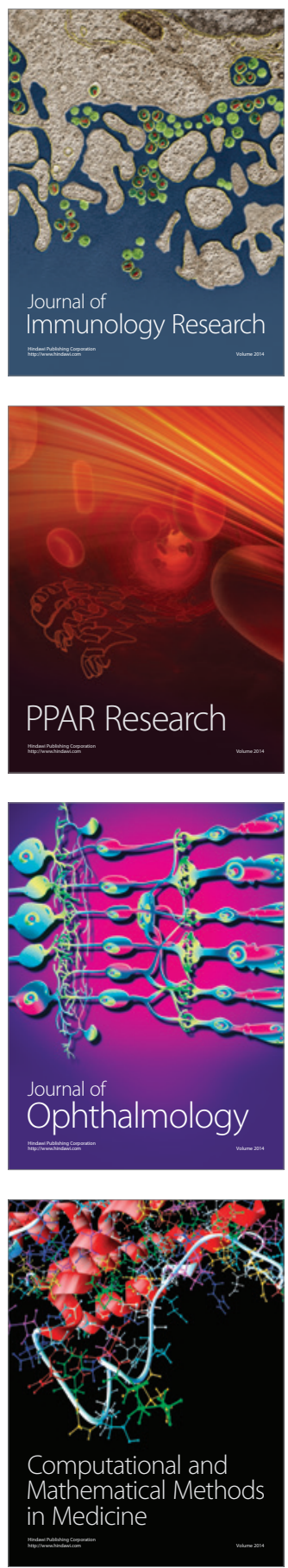

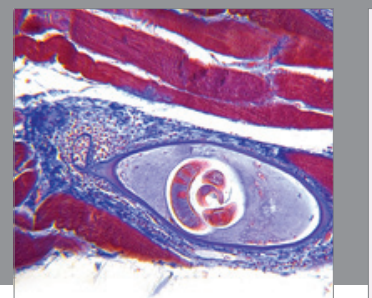

Gastroenterology

Research and Practice
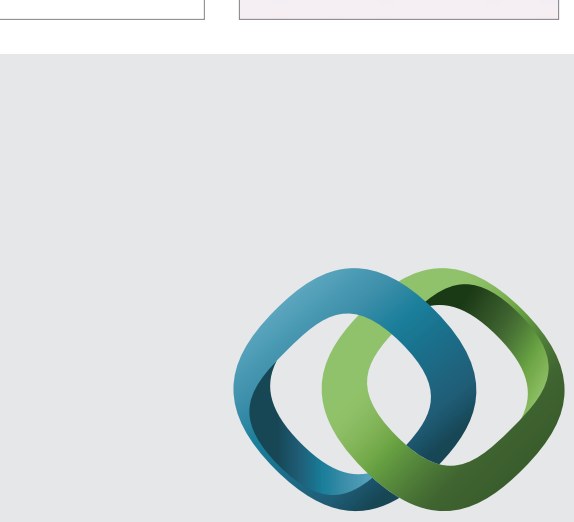

\section{Hindawi}

Submit your manuscripts at

http://www.hindawi.com
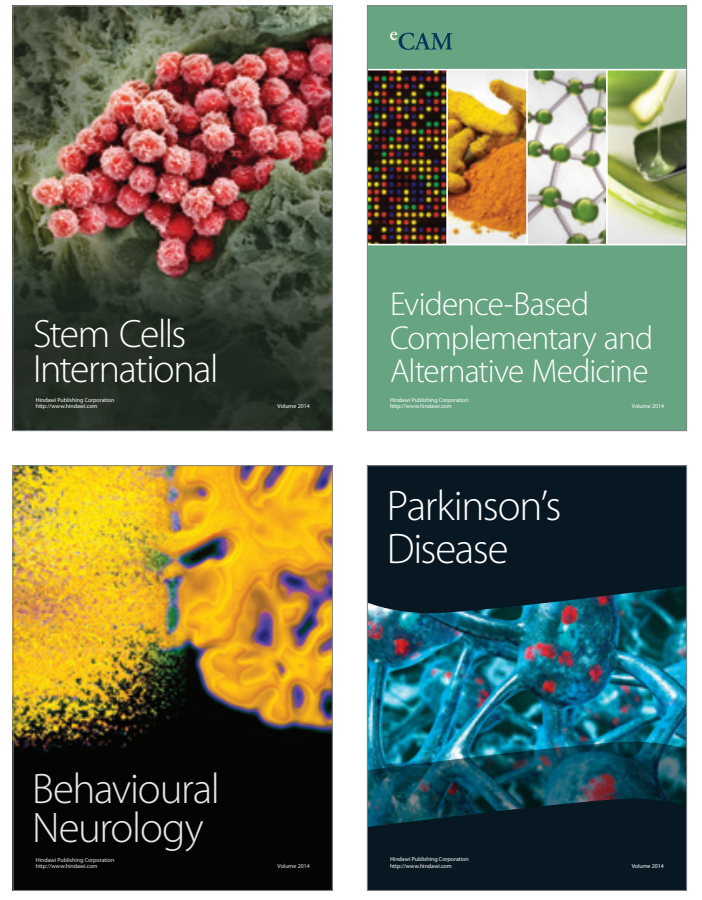
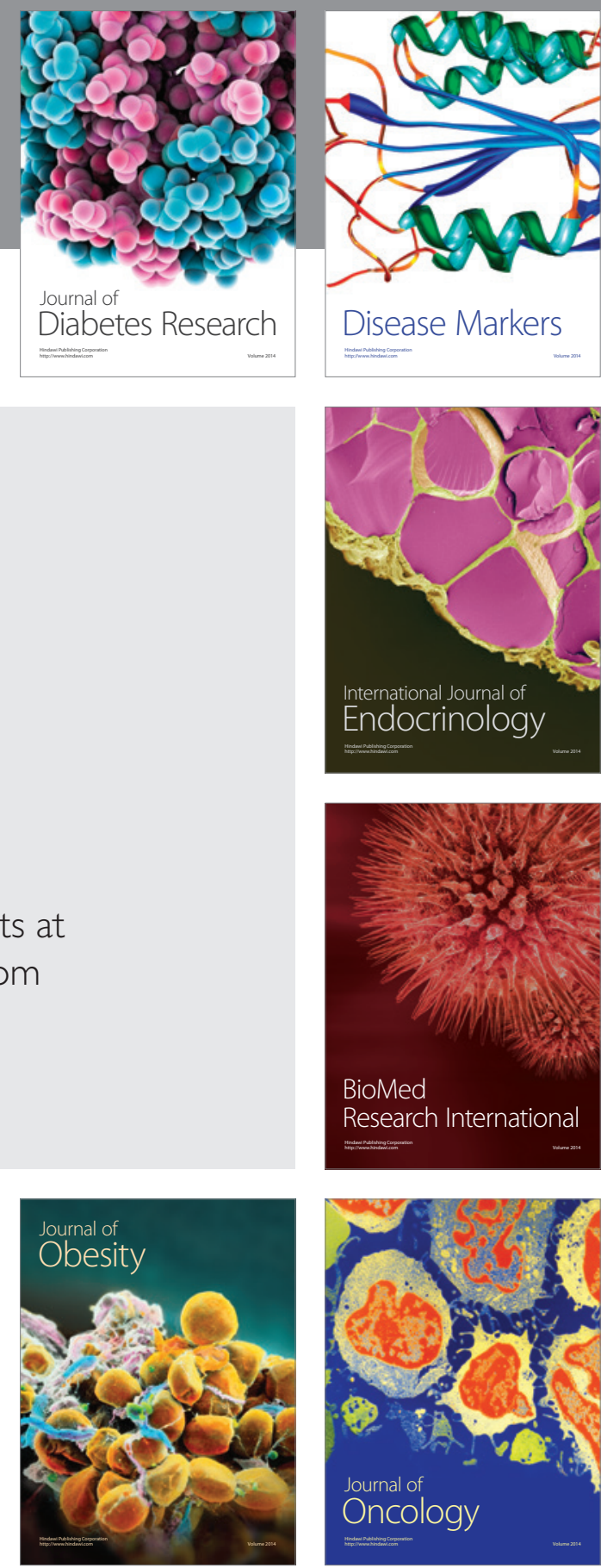

Disease Markers
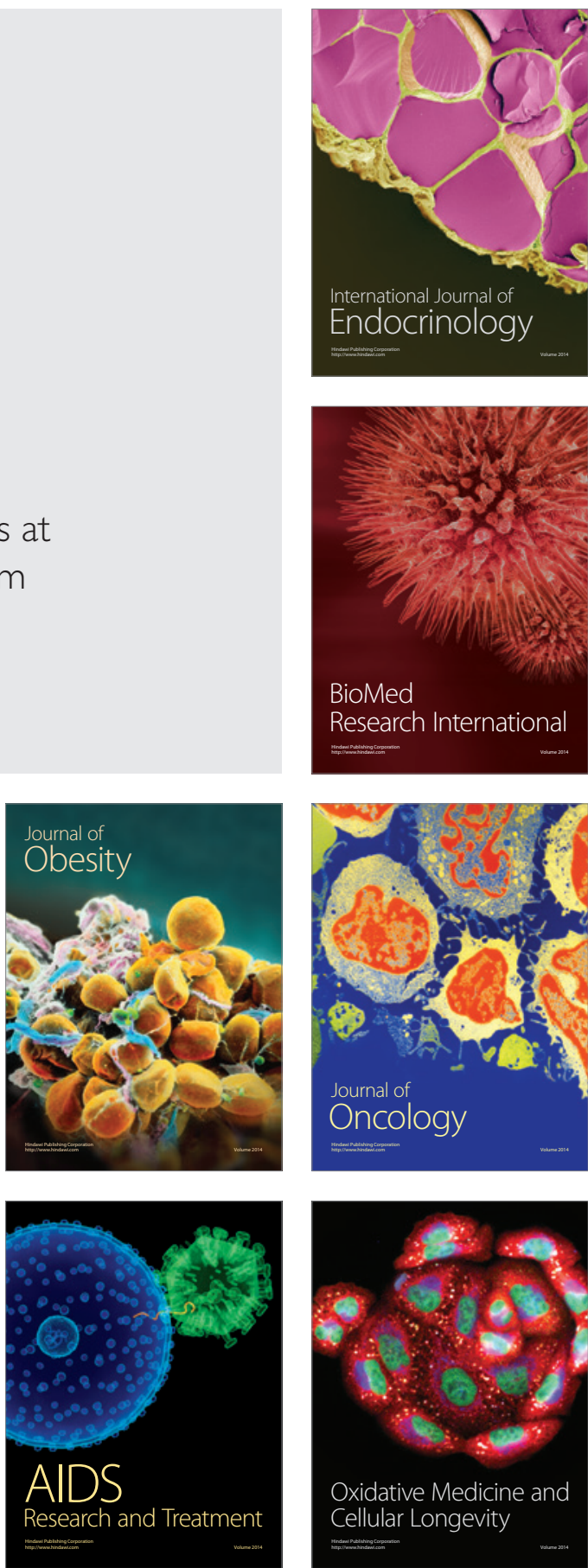